Kristin N. Varhaug, MD

Christian Barro, MD

Kjetil Bjørnevik, MD

Kjell-Morten Myhr, MD,

$\mathrm{PhD}$

Øivind Torkildsen, MD,

$\mathrm{PhD}$

Stig Wergeland, MD,

$\mathrm{PhD}$

Laurence A. Bindoff, MD,

$\mathrm{PhD}$

Jens Kuhle, MD, PhD

Christian Vedeler, MD, $\mathrm{PhD}$

Correspondence to

Dr. Varhaug:

Kristin.nielsen.varhaug@helse-

bergen.no
Supplemental data at Neurology.org/nn

\section{Neurofilament light chain predicts disease activity in relapsing-remitting $\mathrm{MS}$}

OPEN

\section{ABSTRACT}

Objective: To investigate whether serum neurofilament light chain (NF-L) and chitinase 3-like 1 (CHI3L1) predict disease activity in relapsing-remitting MS (RRMS).

Methods: A cohort of 85 patients with RRMS were followed for 2 years $(6$ months without disease-modifying treatment and 18 months with interferon-beta 1a [IFNB-1a]). Expanded Disability Status Scale was scored at baseline and every 6 months thereafter. MRI was performed at baseline and monthly for 9 months and then at months 12 and 24. Serum samples were collected at baseline and months 3, 6, 12, and 24. We analyzed the serum levels of NF-L using a single-molecule array assay and CHI3L1 by ELISA and estimated the association with clinical and MRI disease activity using mixed-effects models.

Results: NF-L levels were significantly higher in patients with new T1 gadolinium-enhancing lesions (37.3 pg/mL, interquartile range [IQR] 25.9-52.4) and new T2 lesions (37.3 pg/mL, IQR 25.1-48.5) compared with those without (28.0 pg/mL, IQR 21.9-36.4, $\beta=1.258, p<0.001$ and $27.7 \mathrm{pg} / \mathrm{mL}$, IQR 21.8-35.1, $\beta=1.251, p<0.001$, respectively). NF-L levels were associated with the presence of T1 gadolinium-enhanced lesions up to 2 months before $(p<0.001)$ and 1 month after $(p=0.009)$ the time of biomarker measurement. NF-L levels fell after initiation of IFNB-1a treatment $(p<0.001)$. Changes in CHI3L1 were not associated with clinical or MRI disease activity or interferon-beta 1a treatment.

Conclusion: Serum NF-L could be a promising biomarker for subclinical MRI activity and treatment response in RRMS. In clinically stable patients, serum NF-L may offer an alternative to MRI monitoring for subclinical disease activity.

ClinicalTrials.gov identifier: NCT00360906. Neurol Neuroimmunol Neuroinflamm 2018;5:e422; doi: $10.1212 / \mathrm{NXI} .0000000000000422$

\section{GLOSSARY}

CHI3L1 = chitinase 3-like 1; $\mathbf{C l}=$ confidence interval; $\mathbf{C I S}=$ clinically isolated syndrome; $\mathbf{C U A}=$ combined unique activity; EDSS = Expanded Disability Status Scale; IFNB-1a = interferon-beta 1a; IQR = interquartile range; $\mathbf{N F - L}=$ neurofilament light chain; $\mathbf{O R}$ = odds ratio; RRMS = relapsing-remitting MS; Simoa = single-molecule array; T1GdE = T1-weighted gadolinium-enhanced.

MS is a chronic inflammatory disease of the CNS in which neuronal damage is present at an early stage. ${ }^{1}$ Due to both unpredictable and heterogeneous disease course and treatment response, biomarkers reflecting these processes are highly sought after.

Neurofilaments are neuron-specific cytoskeletal proteins that can be released following axonal damage. Elevated levels of these proteins have, therefore, been interpreted as reflecting axonal damage and neuronal death in MS, ${ }^{2}$ Alzheimer disease, ${ }^{3}$ fronto-temporal dementia, ${ }^{4}$ and motor neuron diseases. ${ }^{5,6}$ In MS, the neurofilament light chain (NF-L) subunit is considered a potential biomarker for disease activity in CSF. ${ }^{7}$ In addition, correlations have been found between NF-L

From the Department of Neurology (K.N.V., K.B., K.-M.M., Ø.T., S.W., L.A.B., C.V.), Haukeland University Hospital; Department of Clinical Medicine (K.N.V., K.-M.M., Ø.T., S.W., L.A.B., C.V.), University of Bergen, Norway; Neurologic Clinic and Policlinic (C.B., J.K.), Departments of Medicine, Clinical Research and Biomedicine, University Hospital Basel, University of Basel, Switzerland; Department of Global Public Health and Primary Care (K.B.), University of Bergen, Norway; and Norwegian MS-Registry \& Biobank (K.-M.M.).

Funding information and disclosures are provided at the end of the article. Go to Neurology.org/nn for full disclosure forms. The Article Processing Charge was funded by the authors.

This is an open access article distributed under the terms of the Creative Commons Attribution-NonCommercial-NoDerivatives License 4.0 (CC BY-NC-ND), which permits downloading and sharing the work provided it is properly cited. The work cannot be changed in any way or used commercially without permission from the journal. 
and risk of conversion to MS after optic neuritis $^{8}$ and between NF-L and treatment response with immunomodulatory drugs such as fingolimod ${ }^{9,10}$ and natalizumab. ${ }^{11}$ However, a biomarker should preferably be detectable in blood and not in CSF, as lumbar puncture is invasive and not appropriate for repetitive sampling and long-time follow-up. Serum levels of NF-L have been investigated in several studies. Elevated NF-L levels have been found in patients with clinically isolated syndrome (CIS) and relapsing-remitting MS (RRMS) and have been correlated with MRI measures of disease severity and EDSS. ${ }^{12,13}$ However, the methods for analyzing NF-L in serum have varied, and a standardized assay is not yet established. Thus, we wanted to evaluate the potential of NF-L as a serum biomarker using a single-molecule array (Simoa) assay, in the follow-up of patients with RRMS, before and during interferon-beta 1a (IFNB-1a) therapy.

We also included another biomarker chitinase 3-like 1 (CHI3L1) also known as YKL 40. CHI3L1 is a member of the glycoside hydrolase 18 chitinase family but lacks chitinolytic activity. ${ }^{14}$ It is upregulated in numerous chronic inflammatory conditions and is expressed by several cells, including microglia, macrophages, and astrocytes. ${ }^{14}$ In proteomic studies, ${ }^{15,16} \mathrm{CHI} 3 \mathrm{~L} 1$ has been identified as a potential biomarker for MS, and CHI3L1 levels in CSF have been proposed as a prognostic marker for conversion from CIS to MS. ${ }^{8,14,17}$

The aim of this study was to evaluate the potential of NF-L and CHI3L1 as serum

\begin{tabular}{|ll|}
\hline Table $1 \quad \begin{array}{l}\text { Demographic and clinical data of patients with relapsing-remitting MS } \\
\text { at baseline }\end{array}$ & Values \\
Variables & \\
\hline Sex, $\mathrm{n}(\%)$ & $56(65.9)$ \\
\hline Female & $29(34.1)$ \\
Male & $38.9(8.4)$ \\
Mean age at inclusion, y (SD) & $33.0(8.6)$ \\
Mean age at symptom onset, y (SD) & $36.8(9.9)$ \\
Mean age at diagnosis, y (SD) & $1.9(3.1)$ \\
Mean disease duration, $y$ (SD) & $1.89(0.8)$ \\
Mean EDSS (SD) & $2(1.5-2.5)$ \\
Median EDSS (interquartile range) & $1.6(0.7)$ \\
\hline Mean no. of relapses the year before inclusion (SD)
\end{tabular}

EDSS $=$ Expanded Disability Scale Status. biomarkers for clinical use in the follow-up of patients with RRMS.

METHODS Study design and patients. The patient cohort has its origin in a multicenter, randomized, double-blind, placebo-controlled trial to investigate the possible effect of $\omega-3$ fatty acids on disease activity in MS (the OFAMS study) and has previously been described in detail. ${ }^{18}$ Our study comprised 85 patients ( $92 \%$ of the originally included patients), all with RRMS diagnosed according to the McDonald criteria. None were receiving immunotherapy at inclusion. Patients were randomized to supplement with high-dose $\omega-3$ fatty acids or placebo (corn oil), and after 6 months, all patients were additionally treated with subcutaneous $44 \mu \mathrm{g}$ of IFNB-1a 3 times a week. Expanded Disability Status Scale (EDSS) scoring was performed at baseline and months 6, 12, and 24. Relapses were recorded throughout the study period. T2-weighted and T1-weighted gadoliniumenhanced (T1GdE) MRI scans were performed at baseline and monthly thereafter for the first 9 months, then at months 12 and 24. MRI outcome measures were lesions defined as the presence or absence of T1GdE lesions, new or enlarged T2-weighted lesions, or a combination of both (combined unique activity; CUA).

Standard protocol approvals, registrations, and patient consents. The study was approved by the Norwegian Regional Committee for Medical and Health Research Ethics (No: REKno. 005.04), and written informed consent was obtained from all patients (ClinicalTrails.gov NCT00360906).

Serum sampling and analysis. Measurement of serum NF-L and CHI3L1 concentrations was performed on samples drawn at baseline and follow-up at months 3, 6, 12, and 24. Serum samples were stored at $-80^{\circ} \mathrm{C}$.

The concentration of NF-L was determined using a Simoa assay (UmanDiagnostics, Umeå, Sweden). ${ }^{19,20}$ The concentration of CHI3L1 in serum was measured using ELISA according to the manufacturer's protocol (R\&D systems, Minneapolis, MN). Intra- and inter-assay coefficients of variation were below $10 \%$. All serum analyses were performed blinded for patient ID and clinical data.

Missing data. A total of 415 samples were analyzed in 85 patients. One sample (1.2\%) at month 3, 3 at month $12(3.5 \%)$, and 6 at month 24 (7.1\%) were missing for NF-L and CHI3L1 measurements. In addition, another $\mathrm{CH} 13 \mathrm{~L} 1$ sample was missing at month 24. MRI scans from 3 patients were missing at month $3(3.5 \%)$, from 1 patient at month $12(1.2 \%)$ and 3 patients at month $24(3.5 \%)$. Four patients (4.7\%) were without EDSS scores at month 24 .

Statistical analyses. We used mixed-effects models to account for repeated measurements. Linear mixed-effects models with random intercept were used to test for differences in logtransformed levels of NF-L and CHI3L1 by age at inclusion, sex, disease duration, relapses (new relapses or no new relapses), progression in EDSS ( $\geq 1$ EDSS point or $<1$ EDSS point during follow-up), IFNB-1a treatment (treatment or no treatment), and MRI activity (new lesions or no new lesions). The regression coefficients from these models were back-transformed to the original scale. We tested whether a random slope improved the model fit by comparing models with a random slope with models without random slope using likelihood ratio tests. As this did not significantly improve the model fit, we did not include a random slope in the final models. We tested for interaction by a comparing model with an interaction term with a model without an 


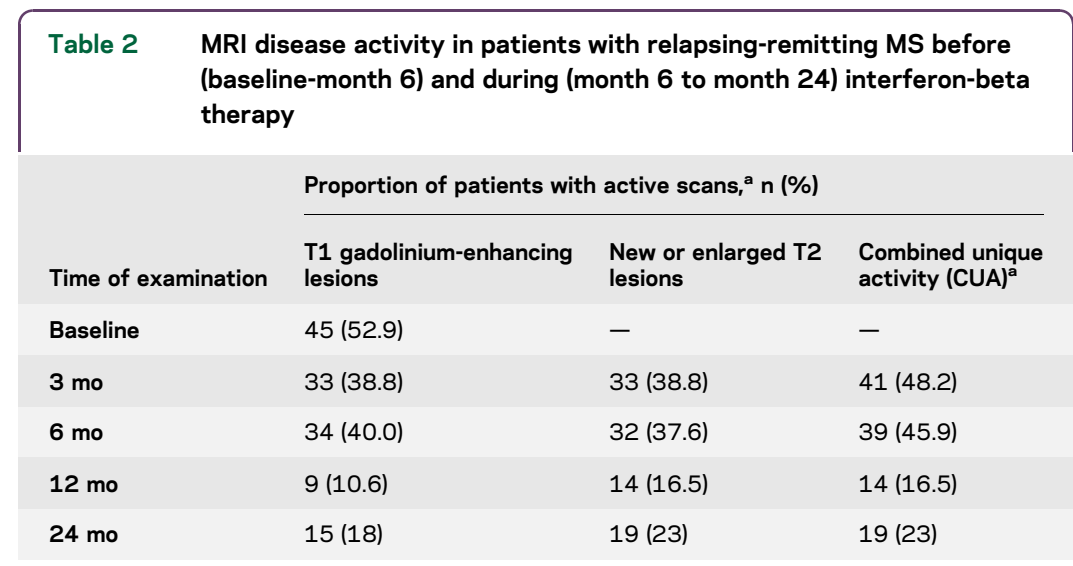

${ }^{a}$ Active scans were defined as the presence of $\mathrm{T} 1$ gadolinium-enhancing lesions, new or enlarged T2 lesions, or a combination of both.

interaction term using the likelihood ratios test. Furthermore, we decomposed NF-L and CHI3L1 into mean levels for each patient during follow-up and deviation from the mean at each time point to estimate between-patient and within-patient effects. We used random intercept logistic regression models to test whether mean protein levels and deviation of the mean were independently associated with MRI activity (new lesions or no new lesions). Last, we created lead and lag variables to test whether NF-L and
CHI3L1 levels were correlated with MRI activity 1, 2, or 3 months before or after the measurement of these proteins. We used the first 9 months of follow-up for these analyses, as MRIs were conducted monthly in this period. As levels of the proteins have been reported to be age dependent, all models were adjusted for age. In addition, we adjusted all models for sex and follow-up time. We tested model assumptions of the mixed-effects models using diagnostic plots. All analyses were performed in Stata 14.1., while figures were made in $\mathrm{R}$ version 3.3.2. A $p$ value of $<0.05$ was considered statistically significant.

RESULTS Patients and MRI activity. Demographic and clinical characteristics of the patients are summarized in table 1. MRI disease activity, defined as patients with active scans, during the study period is shown in table 2.

Association between demographic data and serum NF-L and CHI3L1. Increased NF-L $(p=0.001)$ but not CHI3L1 levels were associated with age at inclusion. Neither NF-L nor CHI3L1 levels were associated with sex.

Association between clinical data and NF-L and CHI3L1. Twenty-six patients experienced disability progression ( $\geq 1$ EDSS point) in the study period,

\section{Figure 1 Levels of neurofilament light chain (NF-L) associated with follow-up time (A) and MRI (B-D)}

A. Serum levels during follow-up

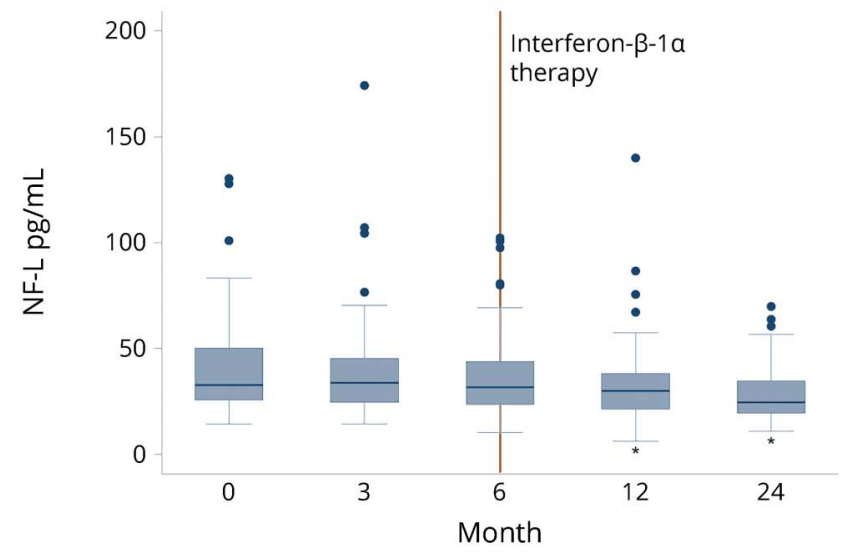

C. T2-weighted lesions

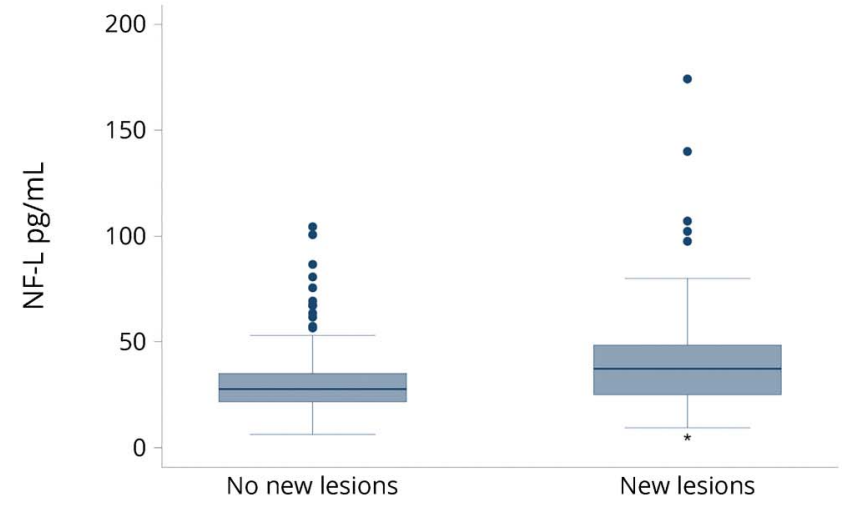

B. T1-weighted GdE lesions

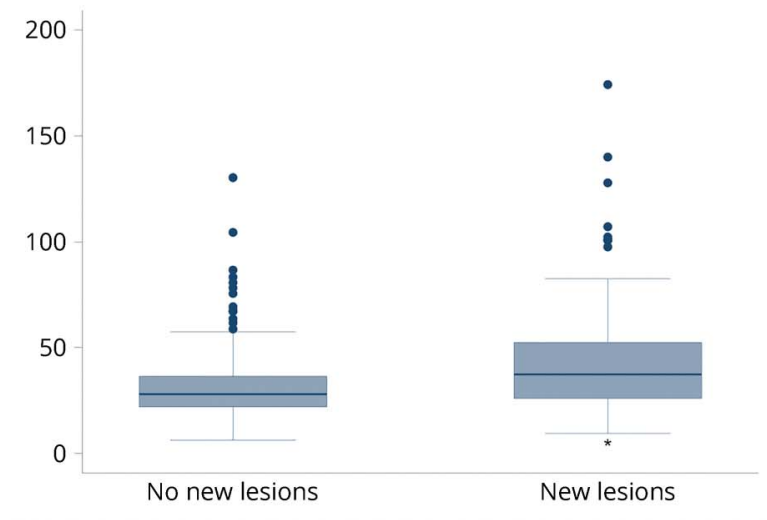

D. Combined unique activity

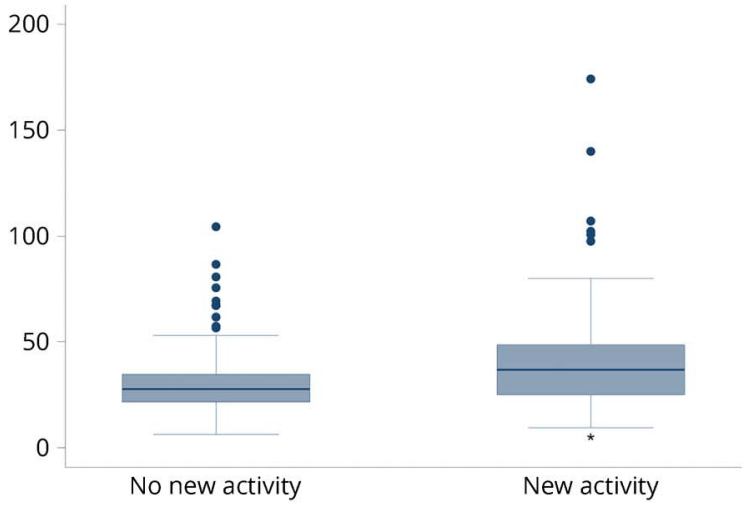

$* p<0.05$ 
and there were a total of 42 relapses in 23 patients. In multivariable linear mixed-effects models, neither NF-L nor CHI3L1 levels were significantly associated with disease duration, relapses, or EDSS progression. Nor was there any association between NF-L or CHI3L1 levels and treatment allocation in the original randomization between $\omega-3$ fatty acids and placebo (the original OFAMS study intervention). The NF-L levels were stable during the first 6 months without treatment, but fell following the initiation of IFNB-1a therapy, and were lower at months 12 and $24(p<0.001$, figure 1A; table e-1 at Neurology.org/nn). CHI3L1 levels were stable during the whole study period, without any influence of IFNB-1a therapy (figure 2A; table e-1).

Association between serum NF-L and CHI3L1 levels and disease activity on MRI during follow-up. Higher NF-L levels were associated with the development of new T1 GdE lesions $(p<0.001)$, new or enlarged T2 lesions $(p<0.001)$, and CUA $(p<0.001)$ (figure 1 , B-D; table e-1) in multivariable analyses adjusting for age, sex, and follow-up time. We examined whether
NF-L levels predicted new MRI-lesions during follow-up on IFNB-1a and found no significant differences in the associations before and after the initiation of treatment ( $p$ for interaction $=0.26,0.20$, and 0.35 for T1-GdE lesions, T2 lesions, and CUA, respectively).

We also examined whether variation in NF-L levels within and between patients was associated with MRI activity and found that both were independently associated with an increased risk of new lesions (table e-2). A within-patient increase of $10 \mathrm{pg} / \mathrm{mL}$ in NF-L levels was associated with increased odds of T1 GdE lesions (odds ratio [OR]: 1.48, 95\% confidence interval $[C I]: 1.15-1.90, p=0.002)$ and new T2 lesions (OR 1.62, 95\% CI 1.22-2.15, $p=0.001$ ).

Finally, we investigated the temporal relationship between NF-L levels and MRI activity. Elevated NF-L was associated with the occurrence of T1 GdE lesions for up to 2 months before and 1 month after the time of biomarker measurement. ( $p<0.001$ and $p=0.009$, respectively). Thus, increased NF-L levels were present for a 3-month period during the development of new lesions as compared to patients without new T1 GdE lesions

Figure 2 Levels of chitinase 3-like 1 (CHI3L1) associated with follow-up time (A) and MRI (B-D)

A. Serum levels during follow-up

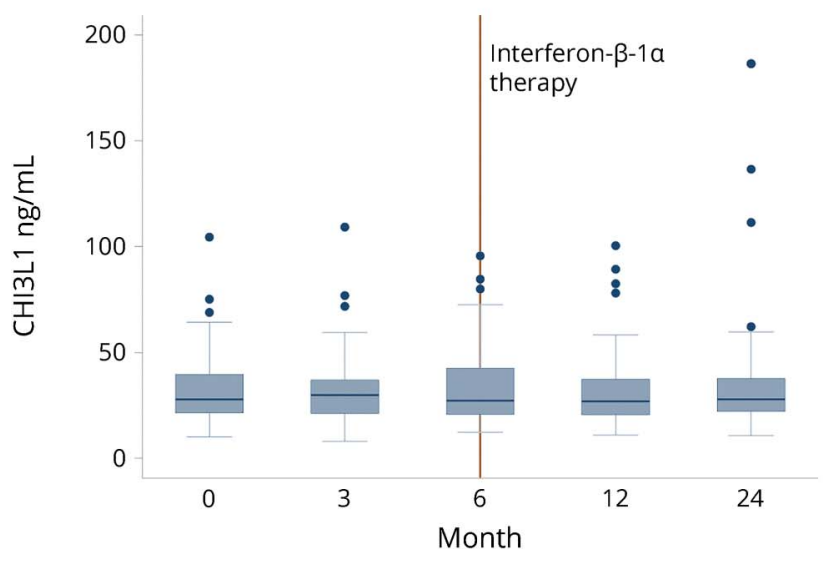

C. T2-weighted lesions

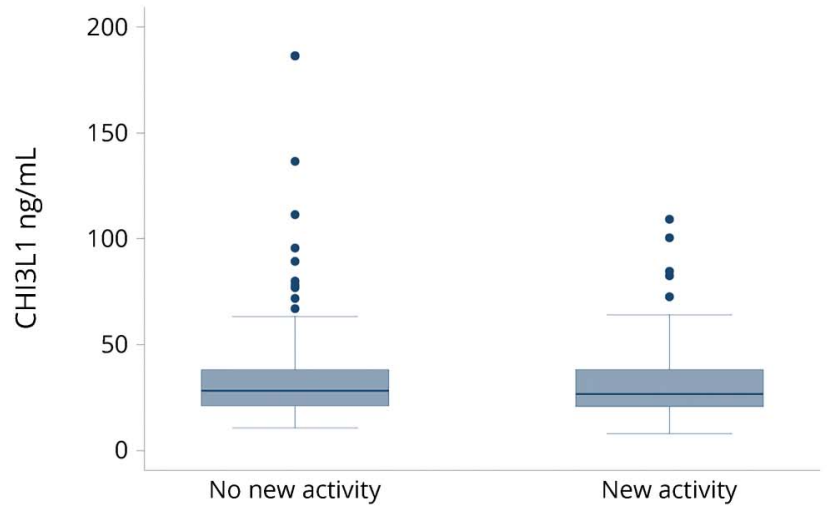

B. T1-weighted GdE lesions

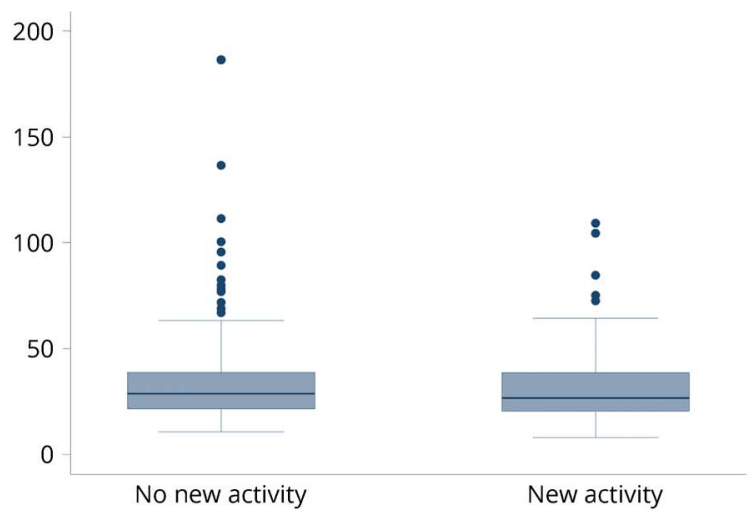

D. Combined unique activity

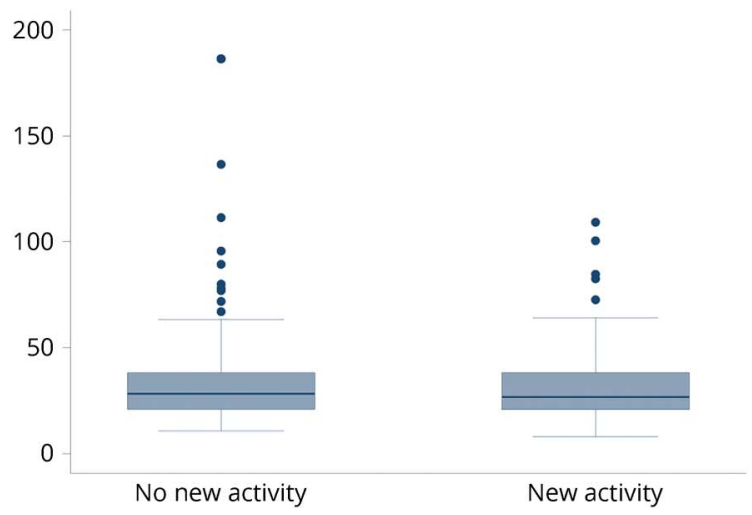


(figure 3). Levels of CHI3L1 showed no association with MRI activity (figure 2, B-D; table e-1).

DISCUSSION We present a large cohort of RRMS in which serum NF-L and CHI3L1 have been evaluated by longitudinal sampling and multiple, clinical, and radiologic assessments. Our most important findings are the associations between serum NF-L and MRI disease activity and the observed reduction in NF-L levels after starting INFB-1a treatment. Due to the longitudinal design of this study with repeated measurements in each patient, we were able to assess whether intraindividual change in NF-L levels were associated with disease activity. As each individual acts as his/her own control in these analyses, they may be less prone to confounding compared with analyses on between-individual changes. We observed that both within and between variation were independently associated with disease activity, adding weight to the evidence linking serum NF-L levels to disease activity.

Our results are similar to those from a study of natalizumab, where CSF NF-L levels decreased to the same level as the healthy controls after 6 or 12 months of treatment, ${ }^{11}$ and to studies looking at CSF NF-L levels and fingolimod treatment. ${ }^{9,10}$ Our results are also in accordance with the lower levels of CSF NF$\mathrm{L}$ in patients treated with mitoxantrone and rituximab. This last study looked, however, at patients with progressive $\mathrm{MS}^{21}$; thus, the results are not directly comparable with ours. Based on our findings, we suggest that NF-L can be regarded as both a biomarker for disease activity and one that reflects the treatment response. NF-L levels are known to be age dependent; thus, the correlation between age and NF-L is not surprising.

No correlation between serum CHI3L1 levels and any clinical or radiologic feature was identified. Furthermore, as has been reported previously, ${ }^{22}$ we found no correlation between age and CHI3L1 levels. Our results showing no significant association with either MRI activity or EDSS score are supported by a recent study that also found no significant differences in CHI3L1 levels and disease activity. ${ }^{23}$ Indeed, this study $^{23}$ suggested that CHI3L1 may reflect the response to IFNB-1a treatment in patients with RRMS. Our study did not support this conclusion.

While we found no association between CHI3L1 and disease activity, levels in CSF have been found to correlate with both GdE lesions and T2 lesions. ${ }^{14}$ This difference could reflect the fact that CSF CHI3L1 is mainly brain derived ${ }^{14}$ and unable to cross the blood-brain barrier sufficiently to give correlating levels in serum. Earlier studies have shown that significant levels of CSF CHI3L1 in patients with CIS were not reproducible in serum. ${ }^{17}$ This probably reflects the finding that the serum concentration of CHI3L1 is eight-fold lower than that in $\mathrm{CSF}^{15}$ and demands greater sensitivity of measurement. Our study confirms that CHI3L1 is not a reliable serum

Figure 3 Relationship between new T1 gadolinium-enhanced lesions and time of biomarker measurement

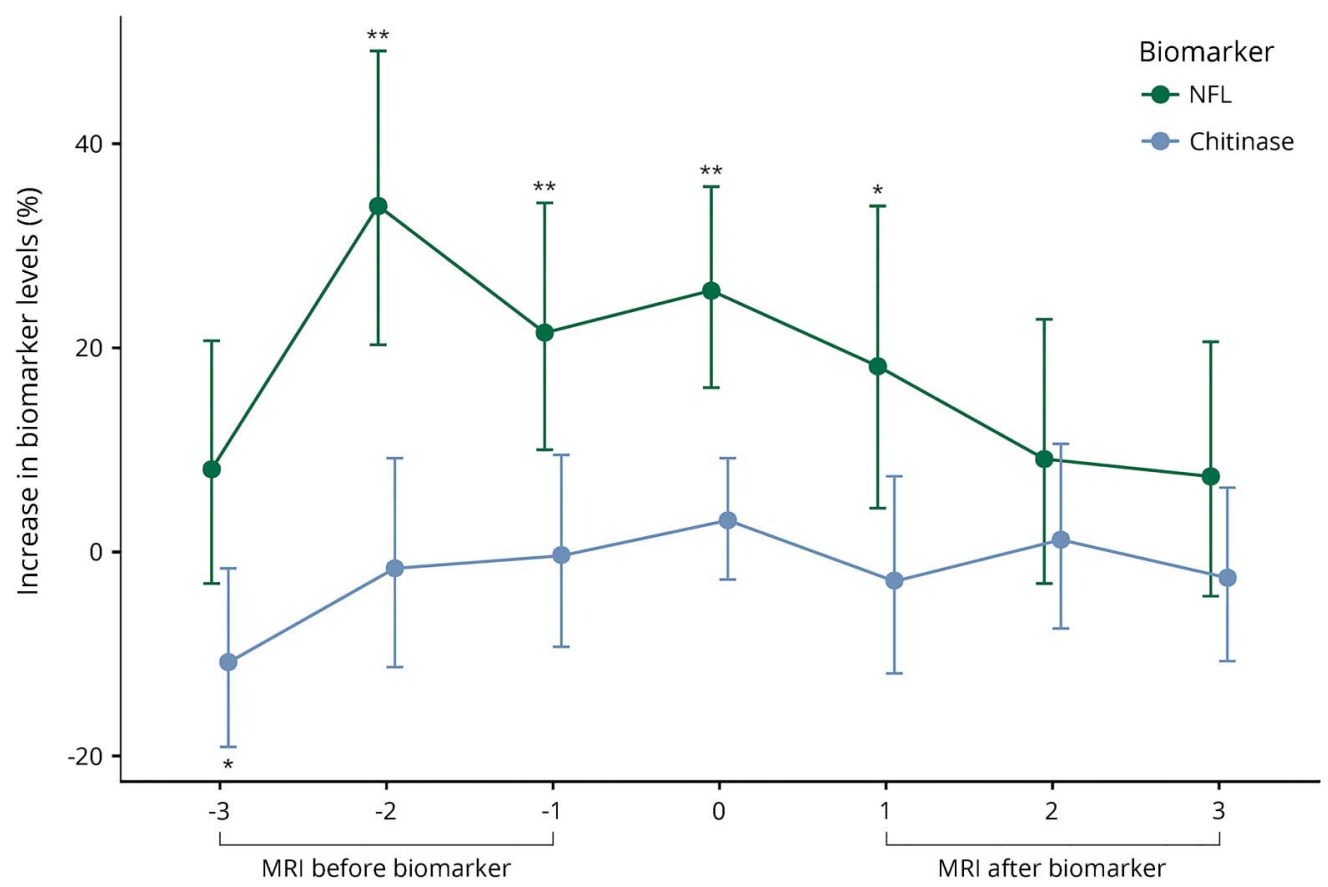

Time (months)

$* p<0.05 ; * * p<0.005$. 
biomarker, despite being a potentially useful CSF biomarker. ${ }^{22}$ The finding of higher plasma concentrations of CHI3L1 in progressive MS than $\mathrm{RRMS}^{24}$ may reflect the presence of other pathogenic pathways than inflammation and this too could indicate that CHI3L1 may not be useful as a prognostic marker for RRMS or treatment response.

Our study has some limitations. First, the MRI scans in our study were conducted more frequently compared with what is done in clinical practice. This may affect the generalizability of our results. Furthermore, the limited follow-up in our study may have affected the power to detect associations between NF-L and clinical outcomes. While most of the patients in our study experienced new lesions during follow-up, only a proportion of the patients experienced relapses or EDSS progression.

Our results indicate that serum NF-L may become a biomarker for the analysis of subclinical MRI activity and treatment response in RRMS. Although the ratio between blood and CSF NF-L levels is between $1 / 30$ and $1 / 70,{ }^{3}$ measurement of NF-L in serum appears sensitive enough to be taken into clinical use. This is in line with recent findings in which NF-L in serum and CSF are highly correlated. ${ }^{25}$ Our findings also suggest that NF-L not only reflects disease activity but also indicates serum NF$\mathrm{L}$ as a promising biomarker in monitoring treatment response. This is also supported by recent studies in which serum NF-L was found to be a sensitive and clinically useful biomarker to monitor tissue damage and effects of therapies in MS. ${ }^{20,25}$ According to our data, rising NF-L levels, with or without clinical symptoms, may act as a trigger for MRI scanning. Therefore, patients with stable clinical course and stable NF-L levels may be spared unnecessary, and costly, MRI scans. This needs to be confirmed in larger prospective patient populations.

\section{AUTHOR CONTRIBUTIONS}

K.N.V. performed the ELISA experiment and contributed to the conception and design of the study, acquisition and analysis of data, and writing and editing of the manuscript. C.V. and K.-M.M. contributed to the original idea and design of the study and writing and editing of the manuscript. Ø.T. and S.W. contributed to the original idea and design of the study and editing of the manuscript. L.A.B. contributed to acquisition of data and writing and editing of the manuscript. K.B. contributed to analysis of data and writing and editing of the manuscript. J.K. and C. B. performed the NF-L measurements and contributed to writing and editing of the manuscript.

\section{ACKNOWLEDGMENT}

The Kristian Gerhard Jebsen Foundation and the Torbjørg Hauges Legacy supported the study. The authors thank the OFAMS study group, especially Antonie Beiske, Harald Hovdal, and Rune Midgard. The authors also thank Liesbeth Kroondijk for excellent technical help.

\section{STUDY FUNDING}

The Kristian Gerhard Jebsen Foundation and the Torbjørg Hauges Leg acy supported the study.

\section{DISCLOSURE}

K.N. Varhaug reports no disclosures. C. Barro has received travel support from Teva and Novartis. K. Bjørnevik served on the scientific advisory board of Biogen and served as an editorial board member for Frontiers in Neurology. K.-M. Myhr reports unrestricted grants and/or scientific advisory board or speaker honoraria from Almirall, Biogen, Genzyme, Merck, Novartis, Sanofi-Aventis, Pronova, Norweigen MS Society, and Roche outside the submitted work. $\varnothing$. Torkildsen served on the scientific advisory board of Biogen, Sanofi-Aventis, and Merck. S. Wergeland received speaker honoraria from Biogen, Novartis, Norweigen MS Society, and Sanofi-Aventis. L.A. Bindoff served on the medical adjudication committee of Stealth Biotherapeutics; served on the editorial board of Neuromuscular diseases; was employed by Stealth Biotherapeutics; and received research support from NFR. J. Kuhle reports that Dr Kuhle's institution (University Hospital Basel) received and used the following exclusively for research support: consulting fees from Novartis, Protagen AG, Roche, and Teva; speaker fees from the Swiss MS Society, Biogen, Novartis, Roche, and Genzyme; travel expenses from Merck Serono, Novartis, and Roche; and grants from ECTRIMS Research Fellowship Programme, University of Basel, Swiss MS Society, Swiss National Research Foundation, Bayer AG, Biogen, Genzyme, Merck, Novartis, and Roche. C. Vedeler reports no disclosures. Go to Neurology.org/nn for full disclosure forms.

Received August 1, 2017. Accepted in final form September 29, 2017.

\section{REFERENCES}

1. Trapp BD, Peterson J, Ransohoff RM, Rudick R, Mork S, Bo L. Axonal transection in the lesions of multiple sclerosis. N Engl J Med 1998;338:278-285.

2. Lycke JN, Karlsson JE, Andersen O, Rosengren LE. Neurofilament protein in cerebrospinal fluid: a potential marker of activity in multiple sclerosis. J Neurol Neurosurg Psychiatry 1998;64:402-404.

3. Bacioglu M, Maia LF, Preische O, et al. Neurofilament light chain in blood and CSF as marker of disease progression in mouse models and in neurodegenerative diseases. Neuron 2016;91:56-66.

4. Meeter LH, Dopper EG, Jiskoot LC, et al. Neurofilament light chain: a biomarker for genetic frontotemporal dementia. Ann Clin Transl Neurol 2016;3:623-636.

5. Steinacker P, Feneberg E, Weishaupt J, et al. Neurofilaments in the diagnosis of motoneuron diseases: a prospective study on 455 patients. J Neurol Neurosurg Psychiatry 2016;87:12-20.

6. Weydt P, Oeckl P, Huss A, et al. Neurofilament levels as biomarkers in asymptomatic and symptomatic familial amyotrophic lateral sclerosis. Ann Neurol 2016;79: 152-158.

7. Soelberg Sorensen P, Sellebjerg F. Neurofilament in CSFA biomarker of disease activity and long-term prognosis in multiple sclerosis. Mult Scler 2016;22:1112-1113.

8. Modvig S, Degn M, Roed $\mathrm{H}$, et al. Cerebrospinal fluid levels of chitinase 3-like 1 and neurofilament light chain predict multiple sclerosis development and disability after optic neuritis. Mult Scler 2015;21:1761-1770.

9. Kuhle J, Disanto G, Lorscheider J, et al. Fingolimod and CSF neurofilament light chain levels in relapsingremitting multiple sclerosis. Neurology 2015;84:16391643.

10. Novakova L, Axelsson M, Khademi M, et al. Cerebrospinal fluid biomarkers of inflammation and degeneration as measures of fingolimod efficacy in multiple sclerosis. Mult Scler 2017;23:62-71.

11. Gunnarsson M, Malmestrom C, Axelsson M, et al. Axonal damage in relapsing multiple sclerosis is markedly reduced by natalizumab. Ann Neurol 2011;69:83-89. 
12. Kuhle J, Barro C, Disanto G, et al. Serum neurofilament light chain in early relapsing remitting MS is increased and correlates with CSF levels and with MRI measures of disease severity. Mult Scler 2016;22:1550-1559.

13. Disanto G, Adiutori R, Dobson R, et al. Serum neurofilament light chain levels are increased in patients with a clinically isolated syndrome. J Neurol Neurosurg Psychiatry 2016;87:126-129.

14. Canto E, Tintore M, Villar LM, et al. Chitinase 3-like 1: prognostic biomarker in clinically isolated syndromes. Brain 2015;138:918-931.

15. Hinsinger G, Galeotti N, Nabholz N, et al. Chitinase 3like proteins as diagnostic and prognostic biomarkers of multiple sclerosis. Mult Scler 2015;21:1251-1261.

16. Opsahl JA, Vaudel M, Guldbrandsen A, et al. Label-free analysis of human cerebrospinal fluid addressing various normalization strategies and revealing protein groups affected by multiple sclerosis. Proteomics 2016;16:1154-1165.

17. Comabella M, Fernandez M, Martin R, et al. Cerebrospinal fluid chitinase 3-like 1 levels are associated with conversion to multiple sclerosis. Brain 2010;133:1082-1093.

18. Torkildsen O, Wergeland S, Bakke $S$, et al. $\omega-3$ fatty acid treatment in multiple sclerosis (OFAMS Study): a randomized, double-blind, placebo-controlled trial. Arch Neurol 2012;69:1044-1051.
19. Norgren N, Karlsson JE, Rosengren L, Stigbrand T. Monoclonal antibodies selective for low molecular weight neurofilaments. Hybrid Hybridomics 2002;21:53-59.

20. Disanto G, Barro C, Benkert P, et al. Serum Neurofilament light: a biomarker of neuronal damage in multiple sclerosis. Ann Neurol 2017;81:857-870.

21. Axelsson M, Malmestrom C, Gunnarsson M, et al. Immunosuppressive therapy reduces axonal damage in progressive multiple sclerosis. Mult Scler 2014;20:43-50.

22. Novakova L, Axelsson M, Khademi M, et al. Cerebrospinal fluid biomarkers as a measure of disease activity and treatment efficacy in relapsing-remitting multiple sclerosis. J Neurochem 2017;141:296-304.

23. Matute-Blanch C, Rio J, Villar LM, et al. Chitinase 3-like 1 is associated with the response to interferon-beta treatment in multiple sclerosis. J Neuroimmunol 2017;303: 62-65.

24. Canto E, Reverter F, Morcillo-Suarez C, et al. Chitinase 3-like 1 plasma levels are increased in patients with progressive forms of multiple sclerosis. Mult Scler 2012;18: 983-990.

25. Piehl F, Kockum I, Khademi M, et al. Plasma neurofilament light chain levels in patients with MS switching from injectable therapies to fingolimod. Mult Scler Epub 2017 Jun 1 . 


\section{Neurology \\ Neuroimmunology \& Neuroinflammation}

Neurofilament light chain predicts disease activity in relapsing-remitting MS

Kristin N. Varhaug, Christian Barro, Kjetil Bjørnevik, et al.

Neurol Neuroimmunol Neuroinflamm 2018;5;

DOI 10.1212/NXI.0000000000000422

This information is current as of November 29, 2017

\section{Updated Information \& Services}

Supplementary Material

References

Subspecialty Collections

Permissions \& Licensing

Reprints including high resolution figures, can be found at: http://nn.neurology.org/content/5/1/e422.full.html

Supplementary material can be found at: http://nn.neurology.org/content/suppl/2018/01/04/5.1.e422.DC1

This article cites 24 articles, 3 of which you can access for free at: http://nn.neurology.org/content/5/1/e422.full.html\#\#ref-list-1

This article, along with others on similar topics, appears in the following collection(s):

Multiple sclerosis

http://nn.neurology.org//cgi/collection/multiple_sclerosis

Information about reproducing this article in parts (figures,tables) or in its entirety can be found online at:

http://nn.neurology.org/misc/about.xhtml\#permissions

Information about ordering reprints can be found online: http://nn.neurology.org/misc/addir.xhtml\#reprintsus

Neurol Neuroimmunol Neuroinflamm is an official journal of the American Academy of Neurology.

Published since April 2014, it is an open-access, online-only, continuous publication journal. Copyright

Copyright $\odot 2017$ The Author(s). Published by Wolters Kluwer Health, Inc. on behalf of the American

Academy of Neurology.. All rights reserved. Online ISSN: 2332-7812.

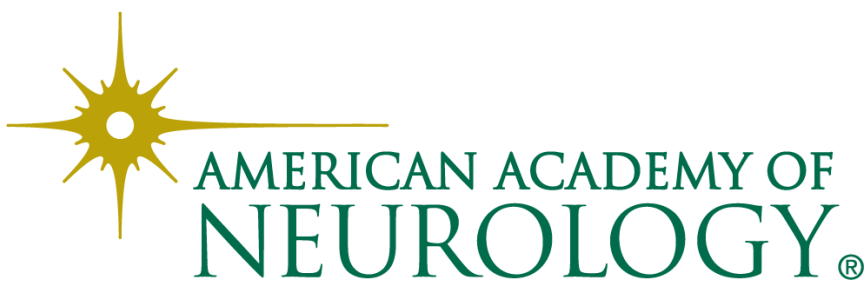

